\title{
The effect of contact torques on porosity of cohesive powders
}

\author{
Guido Bartels ${ }^{1}$, Tamás Unger ${ }^{1,2}$, Dirk Kadau ${ }^{1}$, Dietrich E. Wolf ${ }^{1}$ and János Kertész ${ }^{2}$ *
}

\begin{abstract}
The porosity of uniaxially compacted cohesive powders depends on the applied stress (including gravity). The case, where these stresses are weak, is considered. The compaction results in a porosity which is a function of sliding, rolling and torsion friction. By contact dynamics simulations it is shown that the influences of contact torques (static rolling and torsion friction) on the porosity are significant and approximately additive. The relevance for nano-powder pressure sintering is discussed.
\end{abstract}

Key words. Granular compaction, Cohesive powders, Contact dynamics simulations, Contact torques

\section{1}

\section{Introduction}

The behavior of granular packings under external load is governed by particle properties (e.g. roughness, elasticity, cohesion) as well as by the geometrical structure of the packing (e.g. connectivity, orientations of contacts). When compacting loose granular material, density and connectivity increase until a static state is reached, where the material withstands the external pressure. Such jammed states are currently widely studied $1,2,3$.

For noncohesive materials the porosity in such a jammed state depends essentially on the deformability of the grains under the fixed external load. If the particles

Received: October 30, 2018/ Revised version: date

Guido Bartels, Tamás Unger, Dirk Kadau, Dietrich E. Wolf and János Kertész

Institute of Physics

University Duisburg-Essen

47048 Duisburg, Germany

Institute of Physics

Technical University of Budapest

1111 Budapest, Hungary

PACS number(s): 45.70.Cc, 62.25.+g, 83.10.Rs

* This work was supported by the German Science Foundation (DFG) within SFB 445 "Nano-Particles from the Gas Phase", by the BMBF through grant HUN 02/011 and by Federal Mogul GmbH. We thank Zéno Farkas and Lothar Brendel for useful discussions.

Correspondence to: bartels@comphys.uni-duisburg.de were rigid, the porosity could not be reduced by upscaling the external load (including gravity), but only by shaking 4,5].

However, for cohesive powders, static states with much higher porosity are possible. They are due to stabilization mechanisms, which involve the cohesion force as an intrinsic force scale. Therefore, these states are not stable for arbitrary upscaling of the external load, but only up to a threshold depending on the porosity. Exceeding this threshold leads to further compaction of the powder. In order to avoid confusion we call these static states "blocked" instead of jammed.

This paper considers stabilization mechanisms of blocked states in three dimensional cohesive powders of rigid particles. The relative motion of two solid spheres has six degrees of freedom, three translational characterized by a velocity vector $\boldsymbol{v}$ with one normal component and two tangential components (sliding modes), and three rotational characterized by an angular velocity vector $\boldsymbol{\omega}$, again with one normal component (torsion mode) and two tangential components (rolling modes). If any of these modes are damped or blocked they represent specific dissipation or stabilization mechanisms, respectively. Well known examples are the static and sliding friction: the former stabilizes a contact against small tangential forces (blocking), the latter dissipates kinetic energy (damping) in case of sliding.

All modes of relative motion can be blocked: In addition to static friction also resistance against separation, rolling and torsion must be taken into account, i.e. the contact can exert also cohesion force, normal and tangential torques (i.e. torsion and rolling torques, respectively) in order to inhibit relative motion. To what extent these particle interactions stabilize pores in a cohesive powder is the subject of this paper.

Extending previous work in two dimensions [6], we introduce torsion friction in addition to rolling friction and cohesion into contact dynamics simulations of a uniaxial compression process. Of course, two perfectly rigid spheres, if they existed, would only have a contact point, which could neither exert a rolling nor a torsion torque on the particles in contact. Even the Coulomb-AmontondaVinci friction law would not be justified for such an idealized point contact. By contrast, we consider rigid spheres here only as a geometrical idealization of real particles and do not take their contact areas into account explicitly. Implicitly, however, the finite size of the contacts is responsible for the various kinds of friction we consider. 
It is believed that torsion and rolling resistance are of little importance in noncohesive granular assemblies of spheres, where indeed in statics the contact-torques vanish 7 or are very weak 8 . Our work was motivated rather by powders with grain size smaller than 100nm. The field of nano-powders 9] attracts a lot of scientific and industrial interest due to their different material properties compared to assemblies of larger grains or bulk materials. Many concepts developed for regular granular media can be applied also to nano-powders, but additional aspects have to be taken into account as well, such as strong cohesion (due to van der Waals forces) and sinter-neck formation [10] between the grains which make the question of blocking torsion and rolling modes relevant. Little is known so far about the way in which a sinter-neck resists rolling or torsion. It is plausible, however, that on a sufficiently short time scale (where creep can be neglected) torque thresholds have to be exceeded to break a sinterneck and induce relative motion between the particles. Therefore, lacking well proved contact laws on the nanoscale, we assume the simplest kind of threshold dynamics for sliding, rolling and torsion friction.

\section{2}

\section{Model}

We consider rigid spherical particles of identical sizes, but, as mentioned above, force and also torque transmission is allowed at contacts. The total normal force $F_{\mathrm{n}}$ between two grains has two constituents: first the attractive part, which is a constant cohesion force $F_{\text {coh }}$ and second the force $F_{\text {exc }}$ due to the excluded volume constraint:

$F_{\mathrm{n}}=F_{\text {exc }}-F_{\text {coh }}$,

where the repulsion (attraction) is denoted by positive (negative) sign. Depending on external forces acting on the particles the constraint force $F_{\text {exc }}$ can take any positive value. As a consequence the total normal force can be repulsive $F_{\mathrm{n}}>0$ (arbitrarily strong) or attractive $0>$ $F_{\mathrm{n}}>-F_{\text {coh }}$ as well and is in this latter case limited by the cohesion force. Thus $F_{\mathrm{n}}$ inhibits detachment as long as the pulling is weaker than the cohesion force $F_{\text {coh }}$. If $F_{\text {coh }}$ is exceeded, the contact starts to open, but breaks only when the work done by the pulling force exceeds the cohesion energy ( $F_{\text {coh }}$ times cohesion range). More details of this implementation of cohesion can be found in [11.

The tangential force, as well as the normal and tangential torques are responsible for blocking the sliding, torsion and rolling modes, respectively. Their implementation is described next.

For the tangential contact force the Coulomb friction law has been modified to include the influence of cohesion: If the relative velocity of the two surfaces is zero (sticking contact) the magnitude of the friction force $\left(\left|F_{\mathrm{t}}\right|\right)$ can be any value up to the threshold $F_{\mathrm{t} \text {,max }}$, while in the case of sliding $\left|F_{\mathrm{t}}\right|=F_{\mathrm{t} \text {, max }}$, and its direction is opposite to the relative velocity (Fig. 1). The maximal friction force is proportional to the normal force including $F_{\text {coh }}$ :

$F_{\mathrm{t}, \max }=\mu\left(F_{\mathrm{n}}+F_{\text {coh }}\right)$,

thus the threshold value vanishes when the contact opens $\left(F_{\mathrm{n}}=-F_{\mathrm{coh}}\right)$.

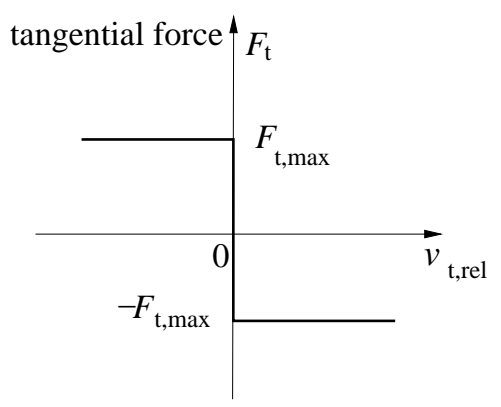

Fig. 1. The graph represents Coulomb's law of friction: A sticking contact (with relative tangential velocity $v_{\mathrm{t}}=0$ ) can bear any tangential force with absolute value up to $F_{\mathrm{t}, \max }$. If sliding occurs $\left(v_{\mathrm{t}} \neq 0\right)$ the magnitude of the tangential force is $F_{\mathrm{t}, \max }$ while its direction is opposite to the sliding velocity.

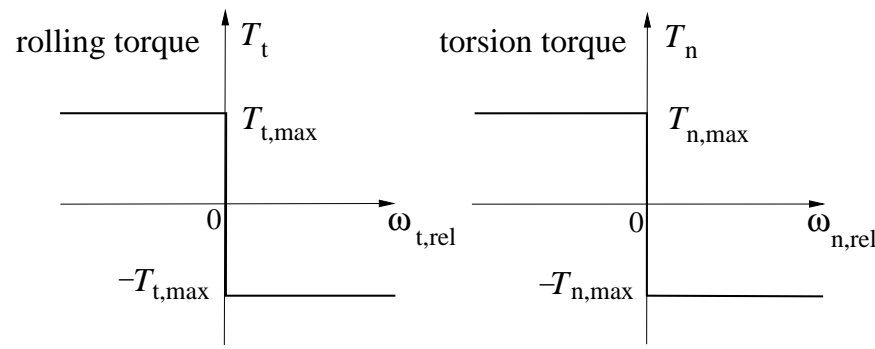

Fig. 2. Contact torques applied to suppress rolling and torsion. The rules are similar to those of the tangential force. The rolling torque (torsion torque) is coupled to the tangential (normal) relative angular velocity $\omega_{\mathrm{t}, \mathrm{rel}}\left(\omega_{\mathrm{n}, \mathrm{rel}}\right)$. For definition of the threshold values $T_{\mathrm{n}, \max }$ and $T_{\mathrm{t}, \max }$ see the text.

The contact laws for the normal and tangential torques are chosen in analogy to the Coulomb friction law (see Fig. 2). The threshold values for the torques $T_{\mathrm{n}, \max }$ and $T_{\mathrm{t}, \max }$ are defined as force times length, where the force-scale is again given by $\left(F_{\mathrm{n}}+F_{\mathrm{coh}}\right)$ and the radius of the sinter-neck determines the relevant length-scale (Fig. 3). This length makes sense only if it is below the particle size, and in our study we choose it within the range between zero and the effective radius at the contact: $r_{\mathrm{eff}}=\left(1 / R_{1}+1 / R_{2}\right)^{-1}$, which for two identical spheres is half the particle radius. The maximal normal and tangential torques in the model are thus given by:

$T_{\mathrm{n}, \max }=\mu_{\mathrm{n}}\left(F_{\mathrm{n}}+F_{\mathrm{coh}}\right) r_{\mathrm{eff}}$

$T_{\mathrm{t}, \max }=\mu_{\mathrm{t}}\left(F_{\mathrm{n}}+F_{\mathrm{coh}}\right) r_{\mathrm{eff}}$,

where the dimensionless parameters $\mu_{\mathrm{n}}$ and $\mu_{\mathrm{t}}$ make it possible to control the strength of blocking, similar to the friction coefficient $\mu$ in Eq. (2).

This model is certainly oversimplified, and the physics of nano-particles might be better described by more sophisticated, yet unknown microscopic models. Moreover, in our model rolling, torsion and sliding modes are independent of each other. In general this is not the case as demonstrated for an ordinary disk on a flat surface [8, 12 13, where the coupling between torsion and sliding friction reduces both of them. In this case assuming independence would overestimate friction. If this is generally true, the porosities obtained in our simulations are expected to 


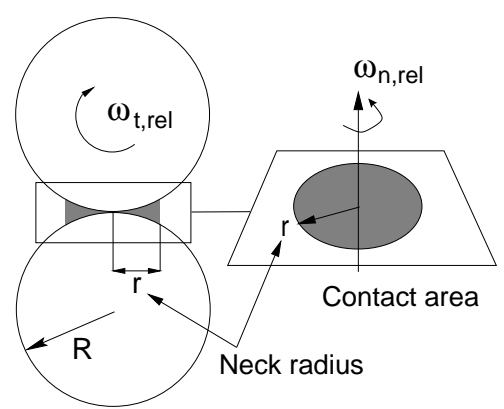

Fig. 3. Schematic figure of a sinter neck between two particles.

be upper bounds. With some caution we can therefore use our simplified model to analyze the effect of rolling and torsion friction on the porosity.

\section{3}

\section{Computer Simulations}

The discrete element simulations presented here were performed with the contact dynamics method 14, 15, 16], where the particles are modeled as non-deformable spheres and the interaction between particles is determined by constraint conditions. The application of constraint forces and torques and an implicit time stepping scheme make this method especially suitable for the implementation of threshold dynamics and for the proper treatment of static forces and torques in blocked or jammed states.

More about our algorithm can be found in 17,18,19. These works contain the description of the basic 3D algorithm for ordinary granular materials, an analysis of the method and its extension to the case of nano-particles.

The system under consideration consists of spherical particles with the same radius $R$. Starting from a low density state the compactibility is measured for various values of $\mu_{\mathrm{t}}$ and $\mu_{\mathrm{n}}$, while the coefficient of friction, $\mu=0.3$, is kept constant.

The initial configuration is prepared by ballistic deposition 20 21, 6]: Particles fall vertically towards a horizontal plane, one by one with randomly chosen $x-y$ coordinates. As soon as a falling particle comes closer than a capture radius $r_{\text {capt }}$ to the deposit or substrate, the contact is established immediately, and the particle sticks irreversibly. Then the next particle is dropped. Here we choose $r_{\text {capt }}=3 R$. As a result one obtains deposits of very low density with tree-like structures. Using this type of initial configuration is motivated by filter processes extracting nano-particles out of a gas flow 22,23.

We simulate the uniaxial compression of this ballistic deposit by a piston moving along the $z$-direction towards the bottom plane, with periodic boundary conditions in $x$ - and $y$-direction. Gravity is neglected. The compression is caused by a constant pressure $F / L_{x} L_{y}$ on the piston (Fig. (4). The simulation ends, when the system reaches a blocked state, i.e. when the piston comes to rest. The system has size $L_{x}=L_{y}=25 R$, contains 1015 particles and the mass of the piston is chosen to $1000 \rho R^{3}$, where $\rho$ denotes the mass density of the particles. The pressure on the piston is $F_{\text {coh }} /\left(400 R^{2}\right)$ in all cases. This is a very weak compacting pressure, as the typical dis-

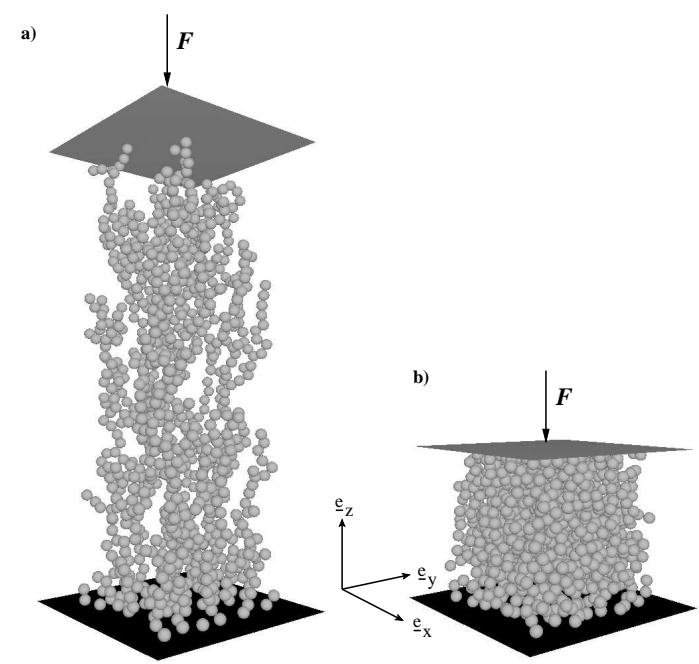

Fig. 4. a) Initial arrangement with 1015 ballistically deposited particles. b) Final configuration of the system compressed by the external force $F$.

tance between branches of the ballistic deposit is of the order of $5 R$, so that the typical load on a single branch, $F_{\text {coh }} 25 / 400=F_{\text {coh }} / 16$, is much smaller than the intrinsic force scale given by the cohesion force $F_{\text {coh }}$.

The mesh of values $\left(\mu_{\mathrm{t}}, \mu_{\mathrm{n}}\right)$, for which we determined the final porosity, is indicated in Fig 5 . Assuming a smooth dependence of the porosity on the friction parameters, we can estimate the error bars from Fig[ 5 without the need to do more than one run for each data point.

\section{4}

\section{Results}

Based on the final position of the piston we measured the porosity $E$ of the blocked states:

$E=\frac{V-V_{\text {grains }}}{V}$

i.e. the relative free-volume in the system (here $V$ denotes the volume between the bottom and the piston). Fig. [5] shows that contact torques have significant effect on the compactification: Whereas without rolling and torsion friction the final porosity is $E_{0} \approx 54 \%$ for the weak compaction pressure we considered, porosities as high as $82 \%$ are stable for $\mu_{\mathrm{t}}=\mu_{\mathrm{n}}=1$.

The porosity added due to rolling and torsion friction,

$E_{1}\left(\mu_{\mathrm{t}}, \mu_{\mathrm{n}}\right)=E-E_{0}$,

saturates in the region where the coefficients $\mu_{\mathrm{t}}$ and $\mu_{\mathrm{n}}$ are larger than 0.4 . More than $E_{1}(1,1) \approx 28 \%$ cannot be achieved based on the contact torques, not even if one increases the threshold values far beyond the physically meaningful range. The maximum porosity is less than the one of the initial configuration: Suppression of rolling and torsion degrees of freedom alone does not suffice to avoid compaction completely.

Qualitatively, rolling friction alone $\left(\mu_{\mathrm{n}}=0\right)$ as well as torsion friction alone $\left(\mu_{\mathrm{t}}=0\right)$ have similar effects on the porosity. Quantitatively we find, that $E_{1}(1,0) \approx 18 \%$ is 


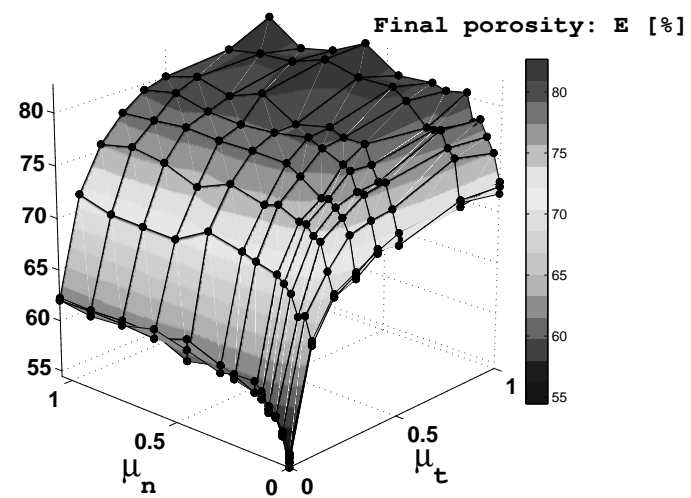

Fig. 5. Final porosity of the compacted system obtained for various values of rolling $\left(\mu_{\mathrm{t}}\right)$ and torsion resistance $\left(\mu_{\mathrm{n}}\right)$.

\begin{tabular}{|cc|ccc|}
\hline$\mu_{\mathrm{t}}$ & $\mu_{\mathrm{n}}$ & $\sigma_{z z}$ & $\sigma_{z z}^{+}$ & $\sigma_{z z}^{-}$ \\
\hline 0.0 & 0.0 & 1.0 & 2.2 & -1.2 \\
0.1 & 0.1 & 1.0 & 1.4 & -0.4 \\
0.3 & 0.3 & 1.0 & 1.1 & -0.1 \\
1.0 & 1.0 & 1.0 & 1.1 & -0.1 \\
\hline 0.0 & 0.3 & 1.0 & 2.2 & -1.2 \\
0.0 & 1.0 & 1.0 & 1.9 & -0.9 \\
\hline 0.3 & 0.0 & 1.0 & 1.2 & -0.2 \\
1.0 & 0.0 & 1.0 & 1.4 & -0.4 \\
\hline
\end{tabular}

Table 1. The influence of the normal and tangential contact torques is shown on the stress transmission along the direction of the uniaxial compression. $\sigma_{z z}^{+}$and $\sigma_{z z}^{-}$are containing only contributions of the compressive and the tensile contacts respectively.

about twice as big as $E_{1}(0,1) \approx 8 \%$. The reason is not clear, but it is intriguing to notice that rolling friction $\mu_{\mathrm{t}}$ suppresses two degrees of freedom, while $\mu_{\mathrm{n}}$ suppresses only one. The ratio $E_{1}(\tilde{\mu}, 0) / E_{1}(0, \tilde{\mu}) \approx 2$ is roughly independent of $\tilde{\mu}$.

An interesting property of the function $E_{1}\left(\mu_{\mathrm{t}}, \mu_{\mathrm{n}}\right)$ is, that it can be very well represented by the following sum:

$$
E_{1}\left(\mu_{\mathrm{t}}, \mu_{\mathrm{n}}\right)=E_{1}\left(\mu_{\mathrm{t}}, 0\right)+E_{1}\left(0, \mu_{\mathrm{n}}\right) .
$$

This shows that rolling and torsion friction contribute independently to the porosity. The difference $|\Delta E|$ between the two sides in Eq. (77) is less than about $3 \%$, and $\Delta E$ fluctuates around zero with no apparent systematics.

Finally we would like to discuss, how the static response of the system to the external load differs with and without contact torques. In the absence of contact torques, strong tensile contact forces appear and seem to be crucial for stabilizing the compressive force lines against buckling in the porous system. This pore stabilization mechanism has also been found in two-dimensional systems [6]. Of course, the contribution of the compressive forces to the macroscopic stress tensor overcompensates the one of the tensile forces in order to balance the external load (table 11).

In the case where contact torques are allowed, rolling and torsion friction already stabilize the force lines against buckling, before significant tensile forces develop. This does not mean that cohesion is superfluous in this case as it is also responsible for the enhanced threshold values of the contact torques (Eq. (3i)).

We measured the macroscopic stress tensor (24):

$\sigma_{i j}=-\frac{1}{V} \sum_{c^{(+)}} F_{i}^{c} l_{j}^{c}-\frac{1}{V} \sum_{c^{(-)}} F_{i}^{c} l_{j}^{c}=\sigma_{i j}^{+}+\sigma_{i j}^{-}$,

separately taking only the contacts under compression $\left(\sigma_{i j}^{+}\right)$or the ones under tension $\left(\sigma_{i j}^{-}\right)$into account. (Here $l_{j}^{c}$ is the $j$ th component of the interstice vector connecting the centers of the particles at contact $c$.) The $z z$ components of the stress tensor are shown in the table (II), where the resulting stress $\sigma_{z z}=F / L_{x} L_{y}$ is of course determined by the pressure on the piston, which is the same for all cases and is taken as unit stress. In the zero-torque case more than twice of the external pressure is provided by the contacts under compression, because a sufficient amount of tension must be allowed in the system in order to stabilize the pores. This internal counter-stress is diminished significantly by rolling friction, whereas torsion friction alone has little effect on the partial stresses $\sigma_{z z}^{+}$ and $\sigma_{z z}^{-}$.

\section{5}

\section{Conclusion}

We presented 3D contact dynamics simulations of cohesive powders and studied the porosity under weak uniaxial compression. We focused on the effect of contact torques which suppress relative torsion and rolling of the adjacent particles. It was found that the presence of contact torques has enormous impact on reducing the final density of the system and in addition the characteristics of the stress transmission is altered: Without torsion and rolling friction strong tensile forces develop and play important role in the mechanical stabilization. These tensile contacts, however, cancel a large part (more than half) of the pressure exerted by compressive forces and only the remaining part is utilized to resolve the external load. This situation is changed by switching on contact torques, which reduce tensile forces significantly in the system. In that case the stress due to the compressive contacts corresponds approximately to the external load and only a small part of this stress is "wasted" to overcompensate tensile forces.

A remarkable feature is the additivity found in the porosity, i.e. the porosity is well represented as the sum of independent contributions of the torsion and rolling friction. Our results suggest that each relative motion mode, if it is suppressed, results in an additional free volume in the system independent of the other modes. Whether this picture holds also for translational degrees of freedom (i.e. altering the Coulomb friction coefficient or the cohesion force) is a subject of further investigation.

\section{References}

1. M. E. Cates, J. P. Wittmer, J. P. Bouchaud, and P. Claudin. Jamming, force chains, and fragile matter. Phys. Rev. Lett., 81:1841, 1998.

2. L. E. Silbert, D. Ertas, Gary S. Grest, T. C. Halsey, and D. Levine. Geometry of frictionless and frictional sphere packings. Phys. Rev. E, 65:031304, 2002. 
3. Hernan A. Makse, Jasna Brujic, and Sam F. Edwards. Statistical mechanics of jammed matter. to appear in "The Physics of Granular Media", eds. H. Hinrichsen and D. E. Wolf (Wiley-VCH, Weinheim, 2004.

4. James B. Knight, Christopher G. Fandrich, Chun Ning Lau, Heinrich M. Jaeger, and Sidney R. Nagel. Density relaxation in a vibrated granular material. Phys. Rev. E, 51:3957, 1995.

5. Emanuele Caglioti, Vittorio Loreto, Hans J. Herrmann, and Mario Nicodemi. A "tetris-like" model for the compaction of dry granular media. Phys. Rev. Lett., 79:1575, 1997.

6. D. Kadau, G. Bartels, L. Brendel, and D. E. Wolf. Pore stabilization in cohesive granular systems. Phase Trans., 76(4-5):315-331, 2003.

7. N. V. Brilliantov and T. Pöschel. Rolling friction of a viscous sphere on a hard plane. Europhys. Lett, 42:511, 1998.

8. Z. Farkas, G. Bartels, Tamás Unger, and D. E. Wolf. Frictional coupling between sliding and spinning motion. Phys. Rev. Lett., 90:248302, 2003.

9. H. Hahn. Unique features and properties of nanostructured materials. Advanced Engineering Materials, 5(5):277, 2003.

10. J. R. Groza. Nanosintering. Nanostruc. Mat., 12:987-992, 1999.

11. D. Kadau, G. Bartels, L. Brendel, and D. E. Wolf. Contact dynamics simulations of compacting cohesive granular systems. Comp. Phys. Comm., 147(1-2):190-193, 2002.

12. K. Voyenli and E. Eriksen. On the motion of an ice hockey puck. American Journal of Physics, 53:1149, 1985.

13. S. Goyal, A. Ruina, and J. Papadopoulos. Planar sliding with dry friction. part 2. dynamics of motion. Wear, 143:331, 1991.

14. J.J. Moreau and P.D. Panagiotopoulos, editors. volume 302 of CISM Courses and Lectures. Springer-Verlag, Wien, New York, 1988.

15. J.J. Moreau. Some numerical methods in multibody dynamics: application to granular materials. Eur $J$ Mech, A/Solids, 13(4):93-114, 1994.

16. M. Jean. The non-smooth contact dynamics method. Comput. Methods Appl. Engrg., 177:235-257, 1999.

17. T. Unger and J. Kertész. The contact dynamics method for granular media. In Modeling of Complex Systems, pages 116-138, Melville, New York, 2003. American Institute of Physics. cond-mat/0211696.

18. T. Unger, L. Brendel, D. E. Wolf, and J. Kertész. Elastic behavior in contact dynamics of rigid particles. Phys. Rev. E, 65, 2002.

19. D. Kadau. Porosität in kohäsiven granularen Pulvern und Nano-Pulvern. PhD thesis, University Duisburg-Essen, 2003.

20. R. Jullien and P. Meakin. Simple three-dimensional models for ballistic deposition with restructuring. Europhys. Lett., 4(12):1385-1390, 1987.

21. P. Meakin and R. Jullien. Ballistic deposition with sticky and non-sticky particles. Physica A, 175(2):211-221, 1991.

22. M. Krafczyk, P. Lehmann, O. Filippova, D. Hänel, and U. Lantermann. Lattice boltzmann simulations of complex multiphase flows. In Multifield Problems, pages 50-57, Berlin, 2000. Springer.

23. O. Filippova and D. Hänel. Lattice boltzmann simulations of gas-particle flow in filters. Comp. and Fluids, 26(7):697712, 1997.

24. J. Christoffersen, M. M. Mehrabadi, and S. Nemat-Nasser. A micromechanical description of granular material behavior. J. Appl. Mech., 48:339, 1981. 